\title{
Magnetic Nanocarriers with Tunable pH Dependence for Controlled Loading and Release of Cationic and Anionic Payloads
}

\author{
Nadja C. Bigall, Alberto Curcio, Manuel Pernia Leal, Andrea Falqui, \\ Domenico Palumberi, Riccardo Di Corato, Ennio Albanesi, Roberto Cingolani, \\ and Teresa Pellegrino*
}

The past two decades have witnessed a growing scientific interest in multifunctional magnetic nanocarriers that are exploitable as tools for diagnosis and treatment of diseases. In the field of materials science, a wide variety of functional nanocarriers (i.e., liposomes, polyelectrolytes, $\mathrm{pH}$-responsive nanogels, etc.) have been developed and exploited as drug or gene carriers. ${ }^{[1-4]}$ To add even more functionality, these nanocarriers have been combined with superparamagnetic nanocrystals into hybrid systems. ${ }^{[5-9]}$ Possible applications of these multiple-task systems include their use as contrast agents for magnetic resonance imaging, as heat mediators for hyperthermia treatment, and as magnetic tools for cell sorting and drug delivery. ${ }^{[10-13]}$ For applications in drug delivery, depending on the type of load, suitable carrier systems should be designed and prepared. Having available magnetic nanocarriers with a suitable surface onto which positively or negatively charged payloads could be loaded would make the system versatile for quite a wide spectrum of applications. To cite a few examples, in drug delivery, attractive electrostatic interactions could be used for adsorbing cationic drugs, such as cationic antimicrobial peptides or positively charged nanoparticles, onto negatively charged carriers. On the other hand, in gene therapy with the aim to insert or inactivate genes in a patient's cell, negatively charged nucleic acids, including plasmids or short-interfering RNA sequences, need to be delivered and could be loaded onto positively charged carriers by a similar concept of electrostatic attraction. ${ }^{[14,15]}$ Moreover, controlled release of charged payloads could be achieved inducing a repulsive electrostatic interaction by inverting the surface charge of the carrier by means of the $\mathrm{pH}$. In particular, the change in zeta potential of the nanosystems entering into the local cell environment, usually coincidences with a change of the electrostatic attraction between the loaded molecules and

Dr. N. C. Bigall, A. Curcio, Dr. M. P. Leal, Dr. A. Falqui, Dr. D. Palumberi, Dr. R. Di Corato, Dr. E. Albanesi, Prof. R. Cingolani, Dr. T. Pellegrino Istituto Italiano di Tecnologia

Via Morego 30, 16163 Genova, Italy

E-mail: teresa.pellegrino@unile.it

Dr. R. Di Corato, Dr. T. Pellegrino

Nanoscience Institute of CNR

National Nanotechnology Laboratory

Via Arnesano, 73100 Lecce, Italy

DOI: 10.1002/adma.201103505 the nanostructure, thus resulting in the release of the molecules. For this purpose versatile systems are required that can undergo a change in their surface charge when penetrating into subcellular compartments. ${ }^{[16]}$ Recently, our group has reported a facile synthesis route for the preparation of magnetic polymer nanobeads by co-precipitation of a polymer and superparamagnetic nanoparticles. ${ }^{[17,18]}$ The resulting nanobeads respond fast to an external magnetic field due to the sum of the magnetic moments of many magnetic nanoparticles included within one nanobead, while keeping superparamagnetic behavior. The carboxylic groups of the polymer backbone confer negative charges to the magnetic beads, thus making our nanostructure not suitable for the interaction with negatively charged molecules. Generally speaking, a more general strategy to tune the surface charge of the nanobeads depending on the $\mathrm{pH}$ would allow adjustment of the electrostatic interaction of the nanobead surface with ionic molecules, resulting in controlled loading and release of various charged payloads.

In order to target these issues, we have developed a new method for fabricating magnetic nanobeads with a tunable $\mathrm{pH}$ responsive surface charge by simply introducing different amino side chains to the polymer used for the bead preparation. First, magnetic nanobeads with isoelectric point at $\mathrm{pH}$ 4.8 were prepared. Positively charged gold nanoparticles were loaded at $\mathrm{pH} 6.5$ onto these magnetic nanobeads and effectively released at $\mathrm{pH}$ 3.5. Second, magnetic nanobeads with an isoelectric point at $\mathrm{pH} 8$ (resulting in a positive zeta potential of the nanobeads in the entire acidic $\mathrm{pH}$ range) were prepared. These beads were used for the loading of (negatively charged) short interfering RNA (siRNA) at pH 6.5. The siRNA loaded nanobeads were applied for the effective down-regulation of the green fluorescent protein (GFP) expression in GFP-transfected HeLa cells, thus proving the siRNA release on cells.

Three different amino-functional side chains were employed to modify poly(maleic anhydride-alt-1-octadecene) (hereafter called polymer I), which was previously used for the synthesis of the magnetic nanobeads (Figure 1A). The monomer unit of polymer I consists of a maleic anhydride group and a hydrophobic hydrocarbon side chain. The anhydride unit offers an anchor point for the functionalization with amino-functional derivates via amide bond formation. Polymer I was modified with three different side chains (Figure 1A): a pyridine derivative (2-(2-pyridyl)ethylamine, polymer II), a primary di-amine derivative (2,2(ethylene dioxy)-bis-(ethylamine), polymer III), 

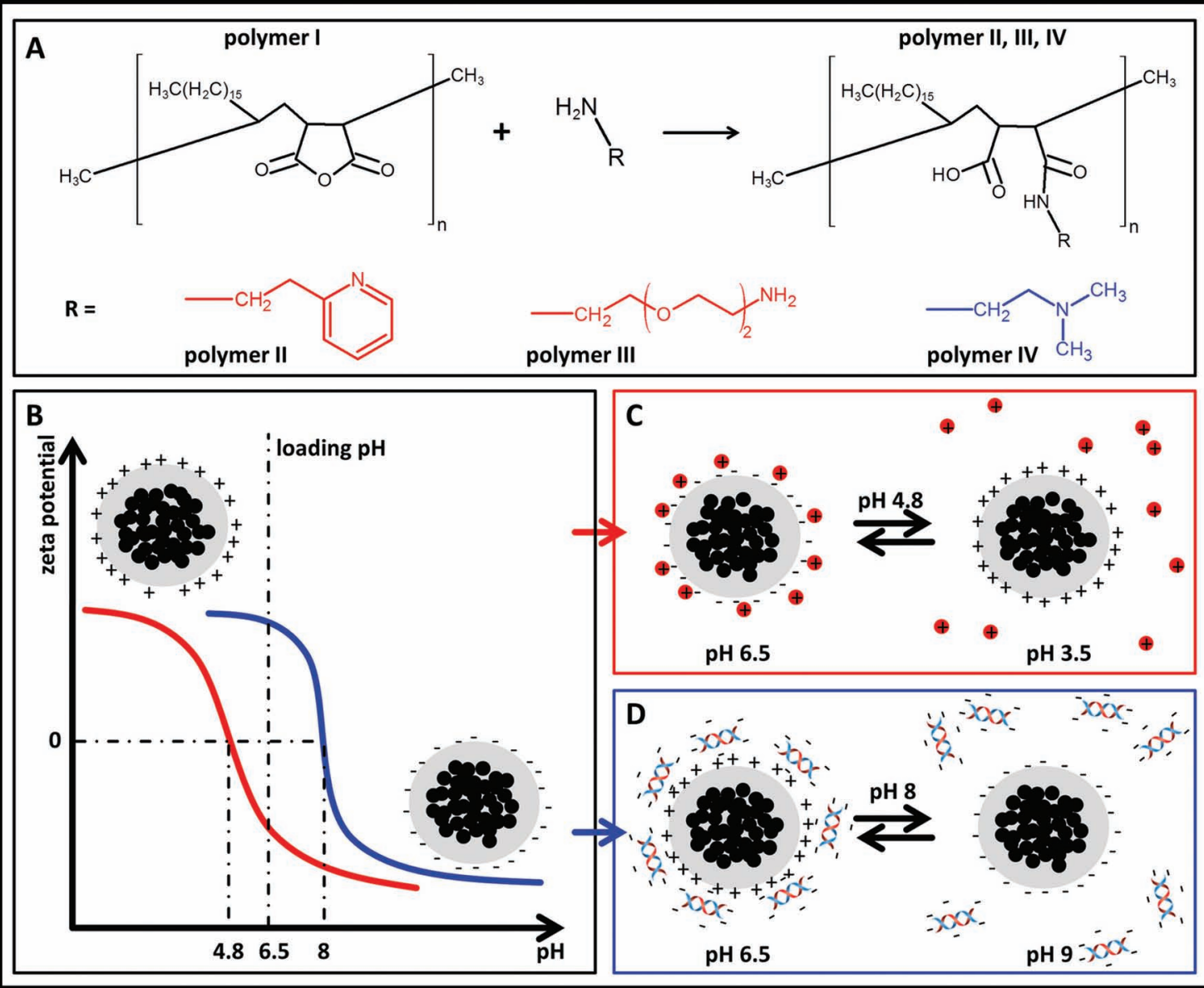

Figure 1. A) Reaction scheme of poly (maleic anhydride-alt-1-octadecene) (polymer I) with amino molecules leading to the $\mathrm{pH}$ responsive polymers having attached pyridine (polymer II), primary amine (polymer III) or ternary amine (polymer IV) side chains. B) Schematic drawing of the different surface charge curves achieved. C) Magnetic nanobeads from polymer II and III follow the red curve and can absorb positively charged gold nanoparticles at pH 6.5 and release them at pH 3.5. D) Magnetic nanobeads from polymer IV exhibit positive surface charge at pH values below 8 . Negatively charged siRNA molecules can be adsorbed onto the nanobeads at $\mathrm{pH} 6.5$, which can be used as RNA carriers on mammalian cells.

and a molecule that introduces ternary amine moieties in the polymer structure ( $N, N$-dimethylethylenediamine, polymer IV). For the polymer functionalization, the respective side chain molecule per each anhydride unit of the polymer I was reacted at a ratio of 0.75 (Supporting Information, S2). ${ }^{[19]}$ The reaction of the polymer backbone with the different amines was confirmed by NMR characterization identifying the amide bond peaks (Supporting Information, S2.1).

For the nanobead preparation, the modified polymer and manganese iron oxide nanoparticles were mixed in tetrahydrofuran (THF), and co-precipitation was induced using a destabilizing agent. Since the different side chains introduced affect the solubility of the modified polymer, for the synthesis of magnetic nanobeads a suitable destabilization solvent had to be properly chosen for each polymer. The different destabilization agents affected the distribution of the inorganic nanoparticles within the beads (Supporting Information, S3.2). ${ }^{[18]}$ The low polydispersity of the bead size from dynamic light scattering (DLS) measurements indicates the stability of the obtained magnetic beads and the absence of agglomerates (Supporting Information, S3.3).
SQUID (superconducting quantum interference device) magnetic measurements proved that all the magnetic nanobeads were superparamagnetic with similar blocking temperatures with respect to the pristine magnetic nanoparticles (Supporting Information, S3.4).

The zeta potentials of the four different types of nanobeads are plotted versus the $\mathrm{pH}$ in Figure 2 (for comparison of hydrodynamic diameters, size distributions, and surface charges of the polymer I to IV nanobeads see Supporting Information, S3.3). In basic solution ( $\mathrm{pH} 9-10$ ), the zeta potential values for the four different nanobead samples are negative (around $-40 \mathrm{mV}$ ). While the nanobeads from polymer I (non-modified polymer) kept a negative surface charge in all the $\mathrm{pH}$ range investigated (from 9 to 2), a tunable switching of the charge from negative to positive values was observed for all the nanobeads obtained from differently modified polymers. The nanobeads from polymer II or III invert their charge from negative to positive values at $\mathrm{pH}$ 4.8. Nanobeads from polymer IV (bearing ternary amines) show an isoelectric point at $\mathrm{pH} 8$, so that they exhibit a positive charge in the whole range from $\mathrm{pH} 8$ to $\mathrm{pH}$ 2. All 


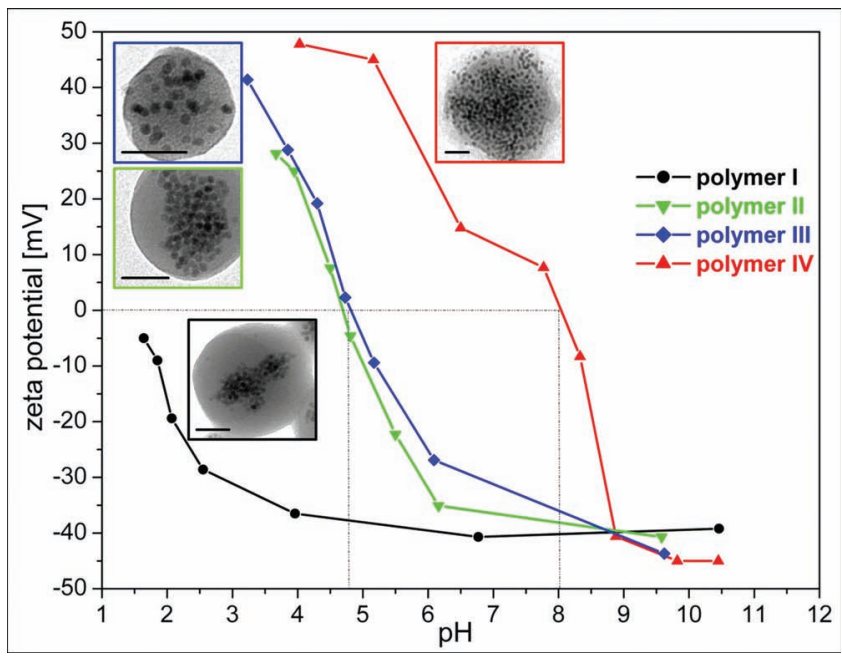

Figure 2. Dependence of the nanobead surface charge on the $\mathrm{pH}$. The zeta potential values are plotted versus the $\mathrm{pH}$. The insets display TEM images of the respective (single) nanobeads each scale bar corresponding to $50 \mathrm{~nm}$.

these results are consistent with the different moieties attached to the original polymer (ternary amines are more basic than primary amines and pyridine-based derivatives, the latter with a $\mathrm{pK}_{\mathrm{a}}$ around 4.5). Therefore, we conclude that the zeta potential values of the nanobeads as a function of the $\mathrm{pH}$, and hence their isoelectric point, can be tuned by choosing the side chains attached to the polymer backbone.

As recently investigated, for sensing or therapeutic purposes it can be interesting to trigger the release of nanoparticles from a magnetic carrier system, including systems releasing optically active nanoparticles or therapeutic nanocrystals, e.g., plasmonic or magnetic nanoparticles. ${ }^{[20,21]}$ Hence, the choice of cationic payload fell to gold nanoparticles (AuNP), since in addition to their characteristic surface plasmon and thermal ablation properties, their contrast in electron microscopy is quite high thus facilitating their visualization. To investigate the suitability of our systems for the goal of $\mathrm{pH}$ mediated loading and release of cationic payloads, the magnetic nanobeads from polymer II and III were investigated for the loading at $\mathrm{pH} 6.5$ of AuNP exhibiting a positive zeta potential. The loading of the polymer III nanobeads was carried out by mixing the two solutions, adjusting the $\mathrm{pH}$ to 6.5 and vortexing the mixture for $1 \mathrm{~h}$. Subsequently, the nanobeads were separated from the solution by magnetic attraction towards a permanent magnet $(0.3 \mathrm{~T})$ placed close to the vial ("magnetic separation"). The nanobeads were redispersed in fresh water at $\mathrm{pH}$ 6.5. By transmission electron microscopy (TEM) characterization the adsorption of AuNP onto the nanobeads surface was confirmed (Figure 3A). Comparing the extinction spectrum of the original AuNP solution with the spectrum of the remaining supernatant after magnetic separation of the nanobeads, it was also found that a significant amount of

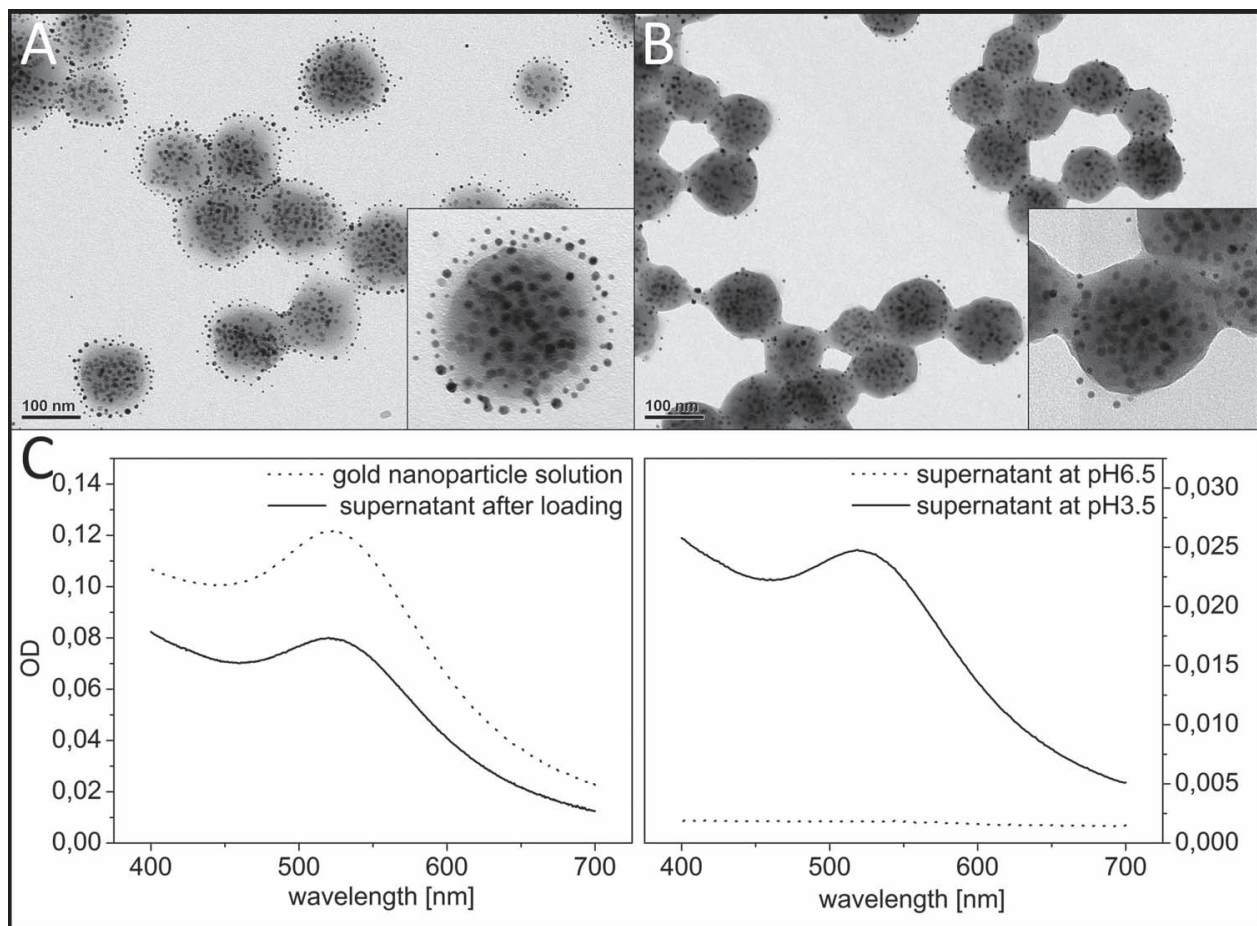

Figure 3. TEM images of polymer III nanobeads A) loaded with gold nanoparticles at $\mathrm{pH} 6.5$ and $\mathrm{B}$ ) after release at $\mathrm{pH} 3.5$. Optical characterization of the gold nanoparticle loading ( $C$, left). Extinction spectra of the gold nanoparticles (without nanobeads) at the starting concentration (dashed) and of the supernatant after magnetically separating the loaded nanobeads from the reaction mixture (full). The signal difference between the dashed line and the full line corresponds to the amount of gold nanoparticles adsorbed onto the nanobeads. Optical characterization of gold nanoparticle release (C, right). Extinction spectra of the supernatants recovered after leaving the gold nanoparticle-loaded nanobeads for 1 day at $\mathrm{pH} 3.5$ (dashed) and of the same sample left for one day at $\mathrm{pH} 6.5$ (full), followed by magnetic separation of the beads. $58 \%$ of the loaded nanoparticles were released after 1 day. 
AuNP was missing in the latter, which corresponded to the fraction of AuNP loaded onto the nanobeads (Figure 3C, left).

For investigating whether the expected release of the payload below pH 4.8 took place, the redispersed nanobeads loaded with AuNP were divided into two portions: one was kept at $\mathrm{pH} 6.5$, while the $\mathrm{pH}$ of the other portion was adjusted to $\mathrm{pH} 3.5$, at which the nanobead zeta potential showed negative values. After shaking the mixture for $24 \mathrm{~h}$, the nanobeads were again separated from the medium by magnetic separation and subsequently redispersed in fresh water. A TEM image of these nanobeads is shown in Figure 3B. A significant release of the AuNP from the nanobeads had occurred. These observations are in good agreement with those from the extinction measurements of the remaining supernatants of the two equally treated samples after the release experiment (Figure 3C, right). The spectrum of the corresponding supernatant of the sample adjusted to $\mathrm{pH} 3.5$ (dashed, Figure 3C right) shows the characteristic extinction of the AuNP, which corresponded to the fraction of AuNP released from the nanobeads. The optical density of the supernatant of the portion of the sample left at $\mathrm{pH} 6.5$ was close to zero in the measured wavelength range, proving that at $\mathrm{pH} 6.5$ no release occurred. By calculating the ratio of the differences in optical densities of the respective data sets shown in Figure 3C (right and left) we calculate that $58 \%$ of the loaded nanoparticles have been released when using polymer III nanocarriers. The change in surface charge of the nanobeads from negative to positive values when switching the $\mathrm{pH}$ from 6.5 to 3.5 likely promotes an electrostatic repulsion of the positively charged AuNP and consequently their release.

The loading and release experiments performed on nanobeads obtained from polymer II provided similar results and are described in detail in the Supporting Information, S4. Hence, we conclude that the nanobeads from polymer II and III are versatile systems for loading and releasing positively charged AuNPs. This principle of $\mathrm{pH}$-induced electrostatic interaction mediated loading and release is expected to be a very general property of the polymer II and III nanobeads. Hence, they might also be interesting carriers for $\mathrm{pH}$ triggered release of cationic drug molecules, which will be the focus of interest in future work.

In the next step, magnetic nanobeads obtained from polymer IV, which exhibit a positive surface charge at $\mathrm{pH}$ 6.5, were investigated to load a negatively charged payload. We chose a siRNA molecule, complementary to a GFP coding sequence (target sequence 5'-GGCAAGCUGACCCUGAAGUUC-3'), labeled at the $5^{\prime}$ of the sense strand with the fluorescence dye Alexa488. The Alexa488-siRNA had a photoluminescence peak at $521 \mathrm{~nm}$ when excited with light of $488 \mathrm{~nm}$ wavelength (Figure 4A). For

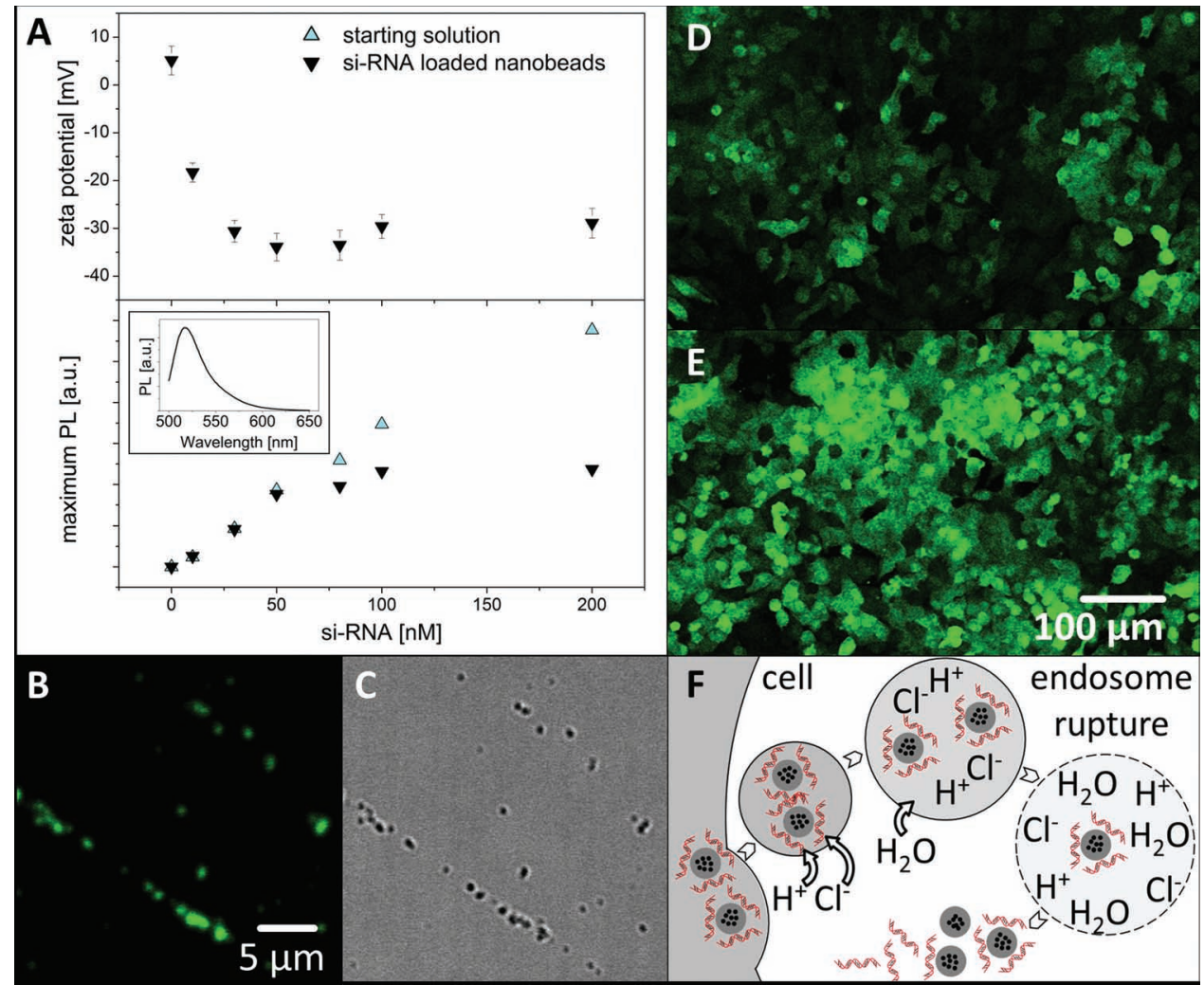

Figure 4. A) Zeta potential values of bare magnetic beads and magnetic beads after separation from Alexa488-siRNA solutions versus the RNA concentration (top). Photoluminescence maxima (bottom) of the initial Alexa488-siRNA solution (light blue) and the signal differences (black) of the initial solution and the supernatant after separation of the nanobeads (corresponding to the amount of Alexa488-siRNA loaded onto the beads) plotted versus the initial Alexa488-siRNA concentration. The inset shows a PL spectrum of an Alexa488-siRNA solution at $488 \mathrm{~nm}$ excitation wavelength. Microscopy images of Alexa488-siRNA loaded nanobeads in B) confocal and C) transmission mode. GFP transfected HeLa cells after $96 \mathrm{~h}$ of incubation D) with siRNA loaded polymer IV nanobeads and E) with nonloaded nanobeads. The significant decrease of the GFP expression when using siRNA loaded nanobeads (D) with respect to the bare nanobeads (E) indicates the suitability of the nanocarriers for gene delivery. F) Cartoon describing the suggested release mechanism via proton-sponge effect. 
the loading, different amounts of Alexa488-siRNA were mixed with a fixed amount of nanobeads at $\mathrm{pH} 6.5$ for $30 \mathrm{~min}$. Subsequently, the nanobeads were separated from the solution by magnetic separation and redissolved in milliQ water at $\mathrm{pH}$ 6.5.

The zeta potential measurements performed on the siRNA loaded nanobeads showed an inversion of the nanobead charge from positive to negative values upon exposure to the Alexa488-siRNA (Figure 4A top), which is a first indication for the Alexa488-siRNA adsorption onto the nanobeads. For Alexa488-siRNA concentrations higher than $50 \mathrm{~nm}$, only small variations of the zeta potential of the Alexa488-siRNA-loaded nanobeads were recorded indicating the coverage saturation of the nanobeads with Alexa488-siRNA sequences.

The amount of Alexa488-siRNA loaded onto the nanobeads was determined by photoluminescence (PL) spectroscopy (Supporting Information, S5.1). As shown in Figure 4A (bottom), for low starting concentrations of Alexa488-siRNA, all the siRNA molecules were adsorbed onto the nanobeads (no significant PL signal was recorded on the supernatant). The saturation occurred at an Alexa488-siRNA concentration of around $50 \mathrm{~nm}$ (the PL signal of the starting solution and the PL signal of the siRNA loaded nanobeads overlap), which is in very good agreement with the observations from the zeta potential measurements. When the initial Alexa488-siRNA concentration was increased even more (at 80, 100, and $200 \mathrm{~nm}$ ), the siRNA loaded on the nanogels stayed constant while the excess of siRNA was left in the supernatant.

By fluorescence microscopy on the nanobeads loaded with Alexa488-siRNA, we could correlate the signal associated to the Alexa488-siRNA in the confocal channel with the dark spots associated to the nanobeads seen in the transmission channel (Figure 4B,C). We were able to exclude the recording of signal from free Alexa488-siRNA and proved that the Alexa488-siRNA is attached to the magnetic nanobeads (see Supporting Information, S5.1 for further information and vertical scan confocal characterization of the nanobeads).

In basic conditions, the Alexa488-siRNA loaded nanobeads were also able to release the siRNA (Supporting Information, S5.2), which proves the versatility of the carrier system. The fact that negatively charged payloads can be released at basic $\mathrm{pH}$ might be of interest for the transport of anionic drugs to the ileum, the intestine section that has neutral or slightly alkaline $\mathrm{pH}$ values.

Since at $\mathrm{pH} 6.5$ the siRNA was observed to be quite stably bound to the polymer IV nanobeads, in the next step, we investigated whether in an in vitro cell culture the siRNA could still effectively be delivered by the cationic magnetic nanobeads. The siRNA loaded nanobeads (the same sequence that did not carry the fluorescent tag) were utilized for knocking down the GFP expression in a HeLa cell line stably transfected with GFP. After incubation under magnetically assisted uptake conditions (Supporting Information, S6.1), the fluorescence of the GFPexpressing cells was analyzed at different times by confocal microscopy. After $96 \mathrm{~h}$, a significant decrease in the green fluorescence signal of the HeLa cells treated with the siRNA loaded nanobeads was recorded with respect to a cell sample treated with nonloaded nanobeads under the same experimental conditions (Figure 4D,E) or with respect to a control cell sample (untreated GFP expressing HeLa cells, see Supporting Information, S6.1 for more information). The quantitative GFP down regulation was also measured by fluorescence activated cell sorting (FACS), and the significant drop of the fluorescence measured in the case of our nanocarriers support their future exploitation in siRNA therapy (Supporting Information, S6.1). ${ }^{[2]}$

The siRNA-loaded nanobeads were found to be taken up through the endocytotic pathway (Supporting Information, S6.2). This suggests the possible siRNA release through a proton sponge effect (Figure $4 \mathrm{~F}$ ); in the acidic endosomal environment the presence of the charged nanocarriers promotes the recall of protons, counter ions and water thus facilitating the endosomal membrane disrupture and consequently the escape of the siRNA from the endosome to the cell cytoplasm where the gene down-regulation occurs. ${ }^{[23,24,25]}$ Remarkably, the polymer IV nanobeads as well as the siRNA loaded polymer IV nanobeads did not show cytotoxicity; the cell viability was around 80\% (Supporting Information, S6.3).

These results are quite promising and suggest future works in order to improve the silencing efficiency by facilitating the endosomal escape pathway, specific cell targeting, or an additional release trigger, e.g., by hyperthermia employing the magnetic properties of the nanobeads.

In conclusion, an amphiphilic polymer has been modified with three different kinds of molecules: a pyridine, a primary amine, and a ternary amine. Nanobeads consisting of superparamagnetic nanoparticles and of the four distinct polymers have been synthesized by a co-precipitation method. The surface charge of the resulting nanobeads at different $\mathrm{pH}$ values resulted to be tunable by the choice of polymer inserted. Nanobeads from the polymers II and III exhibited an inversion of the surface charge at around $\mathrm{pH} 4.8$ and have been shown to effectively load and release positively charged gold nanoparticles depending on the $\mathrm{pH}$ of their environment. Nanobeads from the ternary amine derivative polymer exhibited a positive surface charge for $\mathrm{pH}$ values from 8 to 3. siRNA sequences were proven to effectively adsorb onto these positively charged magnetic nanobeads. In GFP silencing experiments, the nanobeads were shown to be suitable siRNA carriers while maintaining the interfering activity of their payload. These promising results open a variety of application possibilities for multifunctional magnetic nanodevices in the field of gene or drug therapy. In summary, the concept introduced is of great importance for controlled drug release combined with hyperthermia or magnetic resonance imaging (MRI). The combination of the unique magnetic properties of the superparamagnetic nanobeads, their large surface area, and their $\mathrm{pH}$-dependent properties with the simplicity of the fabrication will lead to interesting future applications of these materials in nanomedicine.

\section{Supporting Information}

Supporting Information is available from the Wiley Online Library or from the author.

\section{Acknowledgements}

This work was supported in part by the European project Magnifyco (Contract NMP4-SL-2009-228622). M.P.L. thanks the "Fundación Progreso y Salud" for a postdoctoral fellowship. The authors thank 
Claudio Dalvit for help with the NMR measurements and Liberato Manna for helpful discussions.

Received: September 11, 2011

Published online: November 18, 2011

[1] W. W. Gao, J. M. Chan, O. C. Farokhzad, Mol. Pharm. 2010, 7, 1913.

[2] F. Marcucci, F. Lefoulon, Drug Discovery Today 2004, 9, 219.

[3] S. Ganta, H. Devalapally, A. Shahiwala, M. Amiji, J. Controlled Release. 2008, 126, 187.

[4] G. B. Sukhorukov, A. L. Rogach, M. Garstka, S. Springer, W. J. Parak, A. Munoz-Javier, O. Kreft, A. G. Skirtach, A. S. Susha, Y. Ramaye, R. Palankar, M. Winterhalter, Small 2007, 3, 944.

[5] L. E. Strong, J. L. West, WIREs Nanomed. Nanobiotechnol. 2011, 3, 307.

[6] S. F. Medeiros, A. M. Santos, H. Fessi, A. Elaissari, Int. J. Pharm. 2011, 403, 139.

[7] S. R. Deka, A. Quarta, R. Di Corato, A. Falqui, L. Manna, R. Cingolani, T. Pellegrino, Langmuir 2010, 26, 10315.

[8] O. T. Bruns, H. Ittrich, K. Peldschus, M. G. Kaul, U. I. Tromsdorf, J. Lauterwasser, M. S. Nikolic, B. Mollwitz, M. Merkell, N. C. Bigall, S. Sapra, R. Reimer, H. Hohenberg, H. Weller, A. Eychmuller, G. Adam, U. Beisiegel, J. Heeren, Nat. Nanotechnol. 2009, 4, 193.

[9] L. E. Euliss, S. G. Grancharov, S. O'Brien, T. J. Deming, G. D. Stucky, C. B. Murray, G. A. Held, Nano Lett. 2003, 3, 1489.

[10] F. Gazeau, M. Levy, C. Wilhelm, Nanomedicine 2008, 3, 831.
[11] A. Figuerola, R. Di Corato, L. Manna, T. Pellegrino, Pharmacol. Res. 2010, 62, 126.

[12] H. B. Na, I. C. Song, T. Hyeon, Adv. Mater. 2009, 21, 2133.

[13] S. Mornet, S. Vasseur, F. Grasset, E. Duguet, J. Mater. Chem. 2004, 14, 2161.

[14] M. Sioud, D. R. Sorensen, Biochem. Biophys. Res. Commun. 2003, $312,1220$.

[15] A. Verma, F. Stellacci, Small 2010, 6, 12.

[16] D. Schmaljohann, Adv. Drug Delivery Rev. 2006, 58, 1655.

[17] R. Di Corato, P. Piacenza, M. Musaro, R. Buonsanti, P. D. Cozzoli, M. Zambianchi, G. Barbarella, R. Cingolani, L. Manna, T. Pellegrino, Macromol. Biosci. 2009, 9, 952.

[18] R. Di Corato, N. C. Bigall, A. Ragusa, D. Dorfs, A. Genovese, R. Marotta, L. Manna, T. Pellegrino, ACS Nano 5, 1109.

[19] C. A. J. Lin, R. A. Sperling, J. K. Li, T. Y. Yang, P. Y. Li, M. Zanella, W. H. Chang, W. G. J. Parak, Small 2008, 4, 334.

[20] D. Bartczak, O. L. Muskens, T. M. Millar, T. Sanchez-Elsner, A. G. Kanaras, Nano Lett. 2011, 11, 1358.

[21] K. Hamad-Schifferli, J. J. Schwartz, A. T. Santos, S. G. Zhang, J. M. Jacobson, Nature 2002, 415, 152.

[22] O. Mykhaylyk, O. Zelphati, J. Rosenecker, C. Plank, Curr. Opin. Mol. Ther. 2008, 10, 493.

[23] L. F. Qi, X. H. Gao, ACS Nano 2008, 2, 1403.

[24] A. R. Bayles, H. S. Chahal, D. S. Chahal, C. P. Goldbeck, B. E. Cohen, B. A. Helms, Nano Lett. 2010, 10, 4086.

[25] M. V. Yezhelyev, L. F. Qi, R. M. O'Regan, S. Nie, X. H. Gao, J. Am. Chem. Soc. 2008, 130, 9006. 